\title{
A 12.5-Gb/s Optical Transmitter Using an Auto-power and -modulation Control
}

\author{
Won-Seok Oh* and Kang-Yeob Park \\ Hybrid Signal Processing Research Center, Korea Electronics Technology Institute, \\ Seongnam 463-816, Korea \\ Young-Min Im and Hwe-Kyung Kim \\ System Packaging Research Center, Korea Electronics Technology Institute, Seongnam 463-816, Korea
}

(Received September 15, 2009 : revised November 3, 2009 : accepted November 18, 2009)

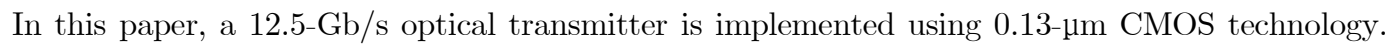
The optical transmitter that we constructed compensates temperature effects of VCSEL (Vertical cavity surface emitting laser) using auto-power control (APC) and auto-modulation control (AMC). An external monitoring photodiode (MPD) detects optical power and modulation. The proposed APC and AMC demonstrate 5 20-mA of bias-current control and 5 20-mA of modulation-current control, respectively. To enhance the bandwidth of the optical transmitter, an active feedback amplifier with negative capacitance compensation is exploited. The whole chip consumes only 140.4-mW of DC power at a single 1.8-V supply under the maximum modulation and bias currents, and occupies the area of $1280-\mu \mathrm{m}$ by $330-\mu \mathrm{m}$ excluding bonding pads.

Keywords: Optical transmitter, Auto-power control, Auto-modulation control, Temperature effect, VCSEL

OCIS codes : : (060.0060) Fiber optics and optical communications; (140.0140) Lasers and laser optics; (140.7260) Vertical cavity surface emitting lasers; (250.3140) Integrated optoelectronic circuits

\section{INTRODUCTION}

Many researchers designing optical transmitters above $10-\mathrm{Gb} / \mathrm{s}$ data rates have implemented their designs using III-V compound semiconductors such as GaAs and InP that provide high bandwidth and good quality passive elements. In low-cost and high volume shorthaul applications around 10-Gb/s (such as LAN (Local area network), MAN (Metropolitan area network), chipto-chip interconnection, and board-to-board interconnection), however, there has been an increasing interest in commercial deep-submicron CMOS technology. It is due to the unique advantages of CMOS technology, such as low power, low cost, good process maturity, and high integration capacity. Therefore, the objective of this paper is to investigate the possibility of implementing a high-speed CMOS optical transmitter for these costsensitive applications [1-2].

For almost semiconductor diodes, the junction voltage at fixed current decreases as the temperature increases.

*Corresponding author: oseok@keti.re.kr

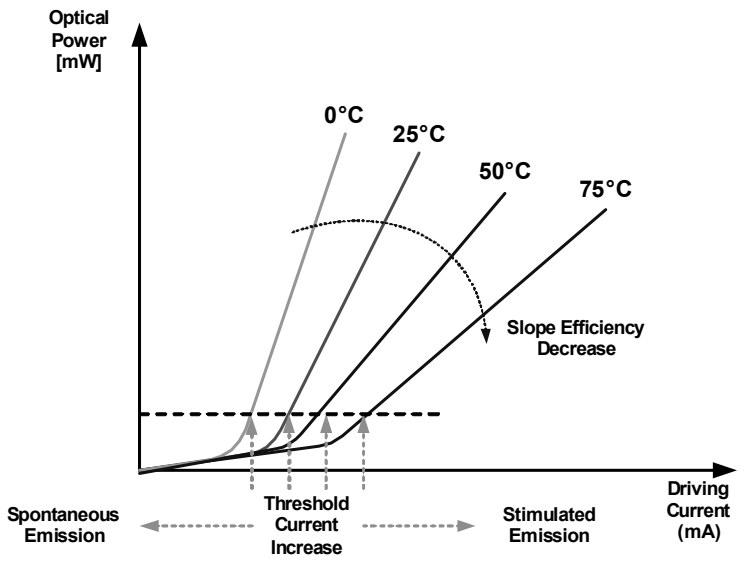

FIG. 1. Conceptual I-P curve of VCSEL with respect to operating temperature.

VCSELs are no exception, with approximately a $-2-\mathrm{mV} /{ }^{\circ} \mathrm{C}$ variation [3]. Fig.1 shows the conceptual I-P curve of the VCSEL according to the operating temperature. The slope efficiency falls off as the temperature increases, thus the modulated optical power decreases and the 
threshold current for the stimulated emission increases. To compensate these temperature-dependent variations, $\mathrm{APC}$ and AMC schemes are essential to maintain constant optical power.

In this paper, a $12.5-\mathrm{Gb} / \mathrm{s}$ optical transmitter using $\mathrm{APC}$ and AMC is designed and implemented in $0.13-\mu \mathrm{m}$ CMOS technology. Section II describes the design details of the optical transmitter, especially APC and AMC schemes. In Section III, the measurement results will be presented and analyzed. Finally, the conclusion is presented in Section IV.

\section{DESIGN DETAILS}

Fig. 2 shows the proposed optical transmitter with APC/AMC function. The optical transmitter which was designed consists of input buffer, pre driver, main driver and APC/AMC blocks. Two VCSELs and a monitoring photodiode (MPD) are externally implemented for the verification APC/AMC schemes. By adding center-tap control voltage (VT) as shown in Fig. 3(a), the input buffer acquires LVDS (Low-voltage differential signaling) and CML (Current-mode logic) compatible input-configuration. In a conventional pre driver, the inductive peaking technique based on an active inductor, a LC-ladder filter network, or a series passive inductor, has been used to enhance the bandwidth. However, they suffer from several problems such

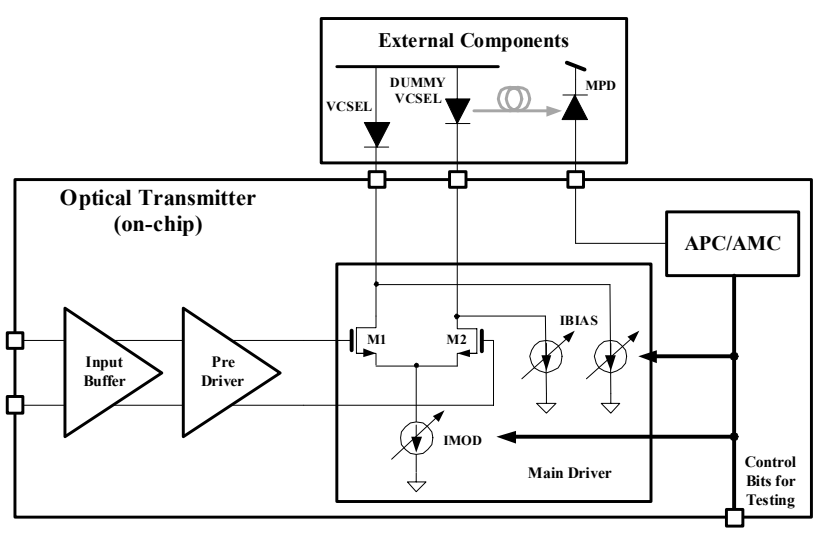

FIG. 2. Proposed optical transmitter with APC/AMC.

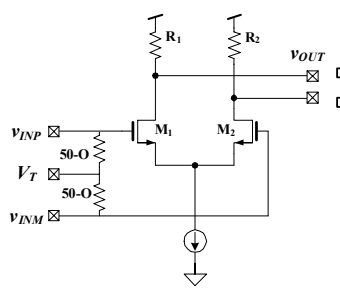

(a)

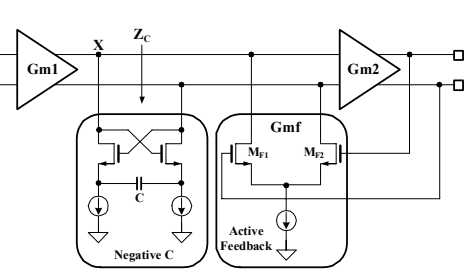

(b)
FIG. 3. Schematics of (a) input buffer and (b) pre driver. as large die area, voltage headroom, and gain fluctuation. To solve these problems, we proposed a pre driver using active feedback and negative impedance compensation (NIC) as shown in Fig. 3(b). Active feedback between two simple common-source amplifiers can improve the gainbandwidth product of the amplifier. A negative transconductance feedback, $G_{m f}$, returns a fraction of the output to the input of $\mathrm{G}_{\mathrm{m} 2}$. Because this architecture doesn't resistively load the trans-impedance stage, the total bandwidth of the amplifier can be enhanced. However, intermediate node (X) between two commonsource stages exhibits three active devices $\left(\mathrm{G}_{\mathrm{m} 1}, \mathrm{G}_{\mathrm{m} 2}\right.$, and $\mathrm{M}_{\mathrm{F} 1}$ ). Thus, this node is very capacitive. To compensate this parasitic capacitance, a NIC stage is added at that point [4].

Fig. 4 shows the block diagram of the main driver with APC/AMC schemes. The main driver drives VCSEL and dummy VCSEL differentially. IBIAS always flows through VCSEL to guarantee that IBIAS always exceeds threshold current for the stimulated emission. IMOD flows through VCSEL according to the incident voltage signal [5].

The detail APC and AMC scheme is presented in Fig. 5. To compensate for temperature effects, on-chip

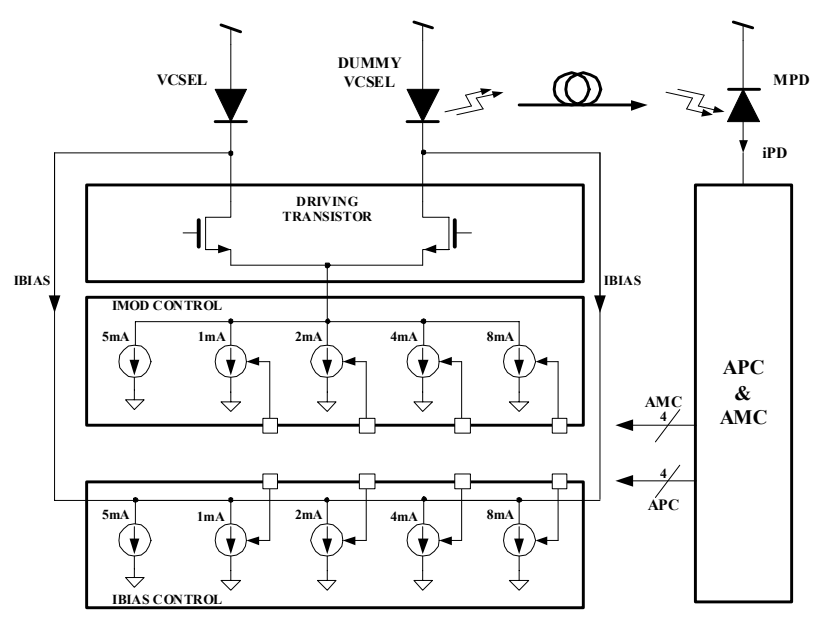

FIG. 4. Main driver with APC/AMC.

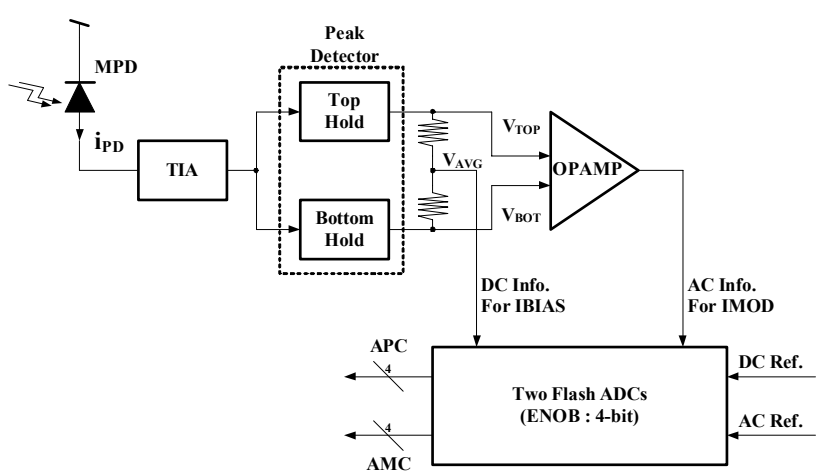

FIG. 5. Detail APC/AMC schemes. 
or off-chip APC and AMC are essentially required. To implement auto or adaptive control schemes, we need to detect the optical power or other corresponding parameters. We exploited monitoring photodiode (MPD) for the detection of optical power. MPD converts the optical power proportionally into electric currents. The output of the MPD includes all AC and DC information. The auto-power control scheme requires only DC information, namely the average optical power, while the auto-modulation control scheme needs only AC information (the modulated power level). The output current of the MPD is too small to be detected. Thus the output of MPD converts and amplifies into a voltage signal through a trans-impedance amplifier (TIA). The peak detector, consisting of top hold and bottom hold circuits, extracts the peak values. The difference of the peak detector outputs carries the AC information for auto-modulation control and the average of the peak detector outputs carries the DC information for auto-power control. Finally, we can get two DC levels including both $\mathrm{AC}$ and $\mathrm{DC}$ information from the optical output by virtue of operational amplifier (OPAMP). And these analog DC levels are converted to the several bits of digital code with the known reference levels for easy control by simple flash-type analog-to-digital converter (ADC), when the information returns to the optical transmitter. We mapped the four digital codes to IBIAS and IMOD in 1-mA resolution per 1-LSB, thus we can control IBIAS and IMOD from default value to (default value $+15-\mathrm{mA}$ ). The default values of IBIAS and IMOD are 5-mA. In other words, we have the controllability $5 \sim 20-\mathrm{mA}$ of IBIAS and $5 \sim 20-\mathrm{mA}$ of IMOD, respectively. In this APC/AMC scheme, by simply adding the digital bits and the number of current source arrays, more precise and wide current control is possible.

\section{MEASUREMENT RESULTS}

The prototype chip has been fabricated in a standard 0.13- $\mu \mathrm{m}$ CMOS technology. The VCSEL driver operates in the frequency ranges from $\mathrm{DC}$ to $12.5-\mathrm{Gb} / \mathrm{s}$ at a $1.8-\mathrm{V}$ supply. The active area excluding bonding pads is $0.43-\mathrm{mm}^{2}$. The chip microphotograph is shown in Fig. 6 .

It is very difficult to measure APC/AMC operation without a chamber for a temperature experiment. Fig. 7 shows the experimental setup to overcome this measurement limitation using a manually controlled optical transmitter and a proposed optical transmitter with APC/AMC. Actually, we fabricated two kinds of chips. One is the optical transmitter with manual bias- and modulation-current control (DUT-1) and the other is the optical transmitter with proposed APC/AMC schemes (DUT-2). By manually controlling bias- and modulationcurrent of DUT-1, we can set up the measurement equivalent of the temperature variation. The inputs of DUT-1 come from a pulse pattern generator. Then, DUT-1 drives the VCSEL with manual control. The MPD detects the different optical power and modulations according to the manual control of DUT-1. Then the proposed APC/AMC performs the automatic control opposing the incident optical power to maintain constant optical power. We measured 4-bits of APC/AMC control codes as a function of the incident optical power,

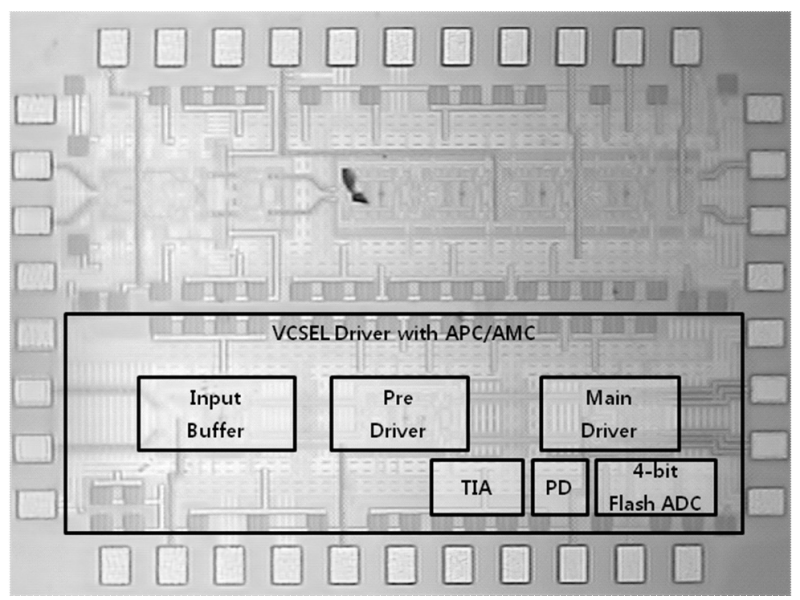

FIG. 6. The chip microphotograph.

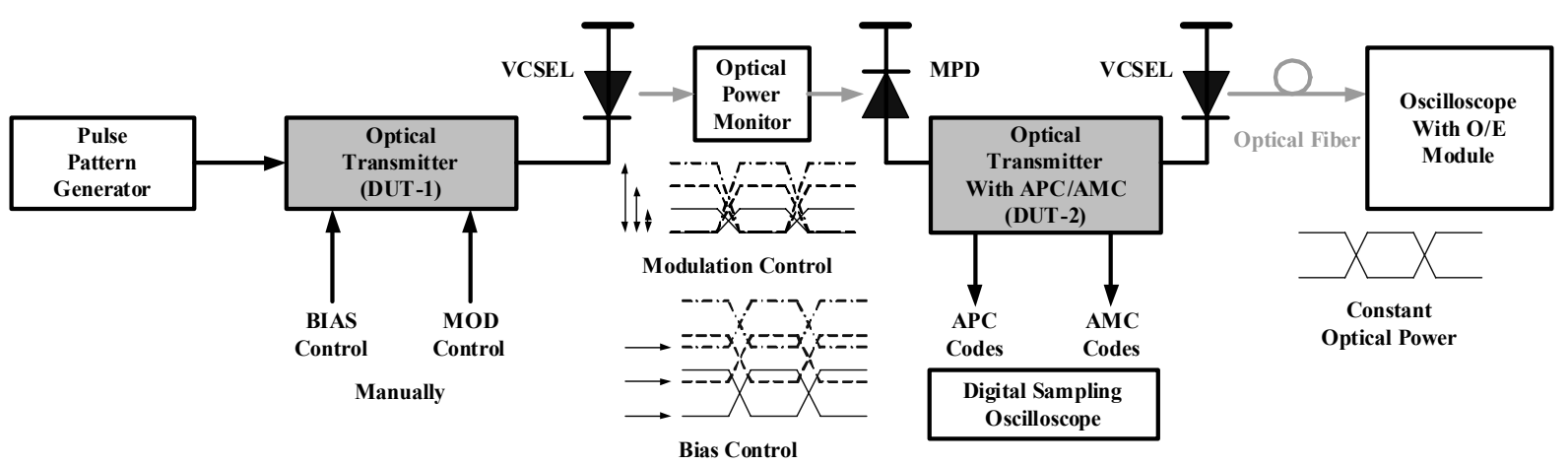

FIG. 7. Measurement setup for APC/AMC. 
as shown in Fig. 8. As the incident optical power increases, APC/AMC control codes decrease linearly.

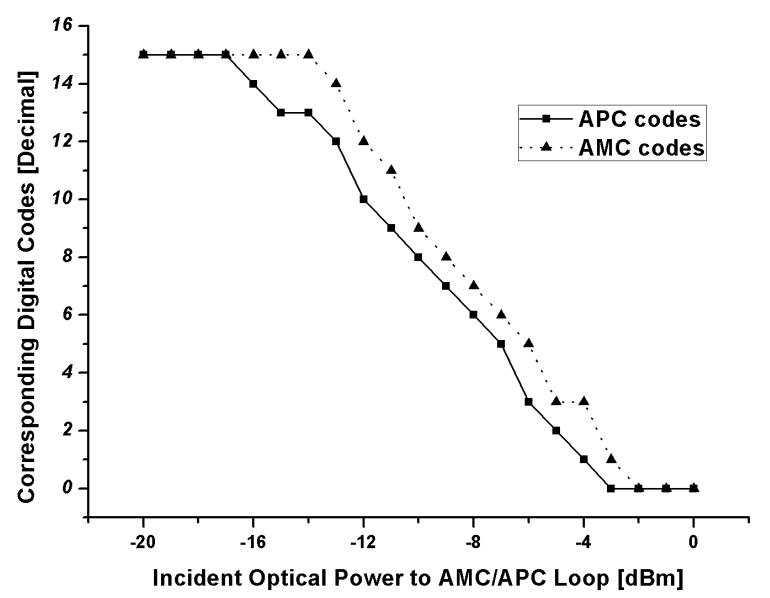

FIG. 8. Measured APC/AMC codes according to the incident optical power.

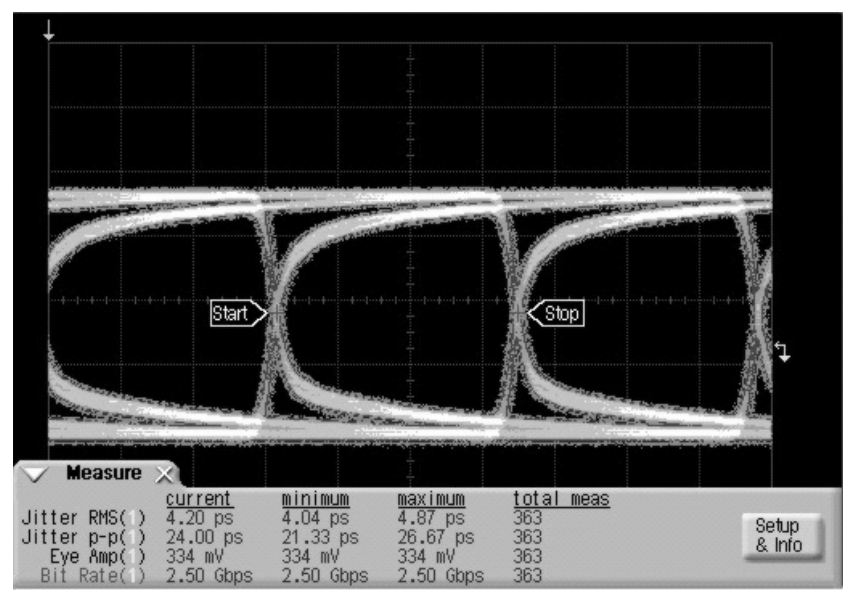

(a)

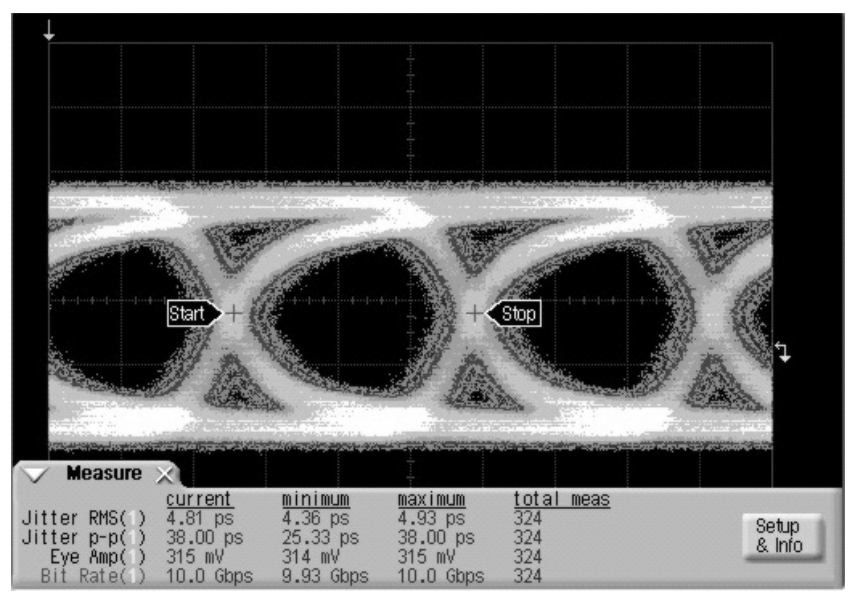

(c)
The decrease of the APC/AMC codes means that the modulation and bias currents of the main driver are decreasing according to the optical power. Finally, the optical transmitter (DUT-2) adaptively drives the VCSEL, and we can show the constant optical eye patterns through the oscilloscope with $\mathrm{O} / \mathrm{E}$ conversion module. Fig. 9 shows the eye patterns for data rates from $2.5-\mathrm{Gb} / \mathrm{s}$ to $12.5-\mathrm{Gb} / \mathrm{s}$.

\section{CONCLUSION}

A $12.5-\mathrm{Gb} / \mathrm{s}$ optical transmitter with proposed APC/AMC loop was designed and implemented in $0.13-\mu \mathrm{m}$ CMOS technology. The proposed APC and AMC loop operates properly with respect to the variation of the incident average and modulated optical power into the monitoring photodiode. APC and AMC loops can control the bias and modulation currents of the optical transmitter by steps of 1-mA over the total range of $15-\mathrm{mA}$. At the circuit level, to enhance the

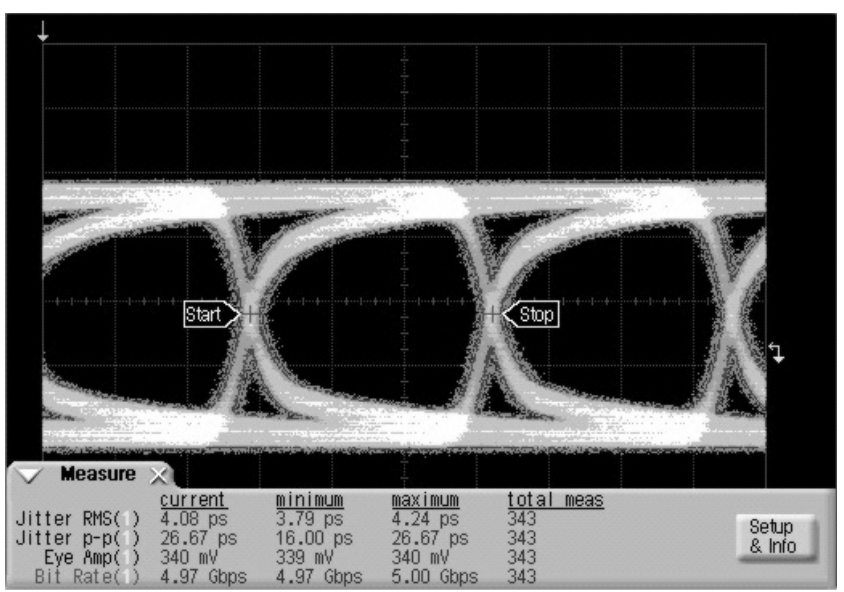

(b)

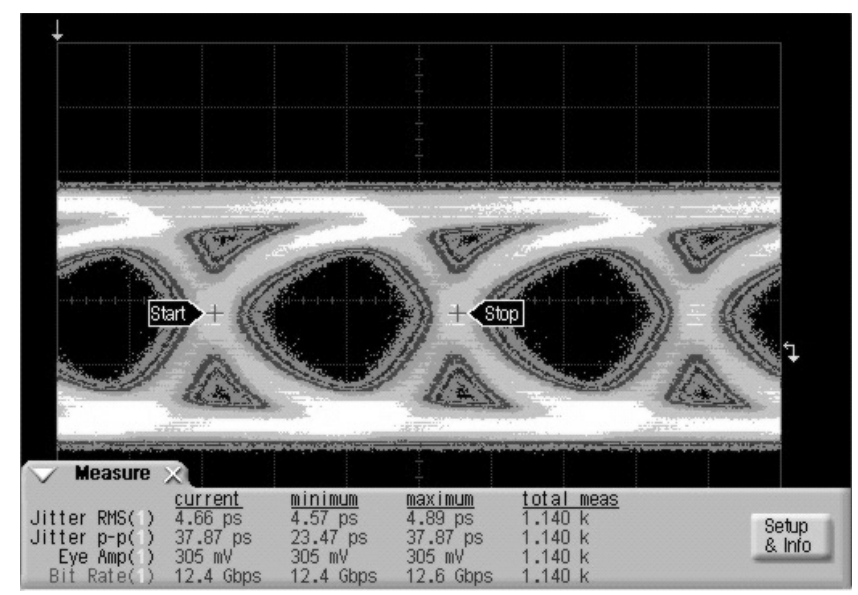

(d)

FIG. 9. Measured eye patterns with respect to the data rates: (a) 2.5-Gb/s, (b) 5-Gb/s, (c) 10-Gb/s, and (d) 12.5-Gb/s. 
bandwidth of the optical transmitter, an active feedback amplifier with negative capacitive compensation was proposed and implemented. The whole chip consumes 78-mA of DC currents at a single $1.8-\mathrm{V}$ supply under the maximum bias (20-mA) and modulation (20-mA) currents. The CMOS optical transmitter with APC/AMC is expected to successfully compensate the temperature effects of the VCSEL and to provide a cost-effective solution for short-haul applications.

\section{REFERENCES}

1. M. S. Lee, B. T. Lee, J. D. Kim, and D. S. Lee, "Fabrication of 2.5 Gbps burst-mode receiver and its full compliance to GPON," J. Opt. Soc. Korea 12, 355-358 (2008).
2. H. S. Kang, M. J. Lee, and W. Y. Choi, “6.25-Gb/s optical receiver using a CMOS-compatible $\mathrm{Si}$ avalanche photodetector," J. Opt. Soc. Korea 12, 217-220 (2008).

3. S. K. Kang, T. W. Lee, H. H. Park, and D. V. Plant, "A novel automatic power control method for multichannel VCSEL driver," in Proc. Pacific Rim Conference on Lasers and Electro-optics (Tokyo, Japan, Jul. 2005), pp. 836-838.

4. R. Tao, M. Berroth, and Z. G. Wang, "Low power 10 Gbit/s VCSEL driver for optical interconnect," Electron. Lett. 39, 1743-1744 (2003).

5. S. Rabii, N. Acharya, P. Chau, J. Dao, A. Feldman, H.J. Liaw, D. Liu, M. Loinaz, M. Luschas, A. Salleh, S. Sheth, S. Sidiropoulos, D. Stark, and S. Verma, "An integrated VCSEL driver for 10Gb ethernet in 0.13-um CMOS," in Proc. IEEE International Conference on Solid-state Circuits (Marriott Hotel, San Francisco, CA, USA, Feb. 2006), pp. 930-939. 